\title{
On shuffling of infinite square-free words
}

\author{
Mike Müller* \\ Institut für Informatik \\ Christian-Albrechts-Universität zu Kiel, Germany \\ mimu@informatik.uni-kiel.de \\ Svetlana Puzynina ${ }^{\dagger}$ \\ LIP, ENS de Lyon \\ Université de Lyon, France \\ and Sobolev Institute of Mathematics \\ Novosibirsk, Russia \\ s.puzynina@gmail.com
}

Submitted: Nov 12, 2014; Accepted: Feb 14, 2015; Published: Mar 6, 2015

Mathematics Subject Classifications: 68R15

\begin{abstract}
In this paper we answer two recent questions from Charlier et al. (2014) and Harju (2013) about self-shuffling words. An infinite word $w$ is called self-shuffling, if $w=\prod_{i=0}^{\infty} U_{i} V_{i}=\prod_{i=0}^{\infty} U_{i}=\prod_{i=0}^{\infty} V_{i}$ for some finite words $U_{i}, V_{i}$. Harju recently asked whether square-free self-shuffling words exist. We answer this question affirmatively. Besides that, we build an infinite word such that no word in its shift orbit closure is self-shuffling, answering positively a question of Charlier et al.
\end{abstract}

Keywords: infinite words, shuffling, square-free words, shift orbit closure, selfshuffling words

\section{Introduction}

A self-shuffling word, a notion which was recently introduced by Charlier et al. [2], is an infinite word that can be reproduced by shuffling it with itself. More formally, given

\footnotetext{
* Supported in part by the DFG under grant 582014 .

${ }^{\dagger}$ Supported by the LABEX MILYON (ANR-10-LABX-0070) of Université de Lyon, within the program "Investissements d'Avenir" (ANR-11-IDEX-0007) operated by the French National Research Agency (ANR).
} 
two infinite words $x, y \in \Sigma^{\omega}$ over a finite alphabet $\Sigma$, we define $\mathscr{S}(x, y) \subseteq \Sigma^{\omega}$ to be the collection of all infinite words $z$ for which there exists a factorization

$$
z=\prod_{i=0}^{\infty} U_{i} V_{i}
$$

with each $U_{i}, V_{i} \in \Sigma^{*}$ and with $x=\prod_{i=0}^{\infty} U_{i}, y=\prod_{i=0}^{\infty} V_{i}$. An infinite word $w \in \Sigma^{\omega}$ is self-shuffling if $w \in \mathscr{S}(w, w)$. Various well-known words, e.g., the Thue-Morse word or the Fibonacci word, were shown to be self-shuffling.

Harju [5] studied shuffles of both finite and infinite square-free words, i.e., words that have no factor of the form $u$ for some non-empty factor $u$. More results on square-free shuffles were obtained independently by Harju and Müller [6], and Currie and Saari [4]. However, the question about the existence of an infinite square-free self-shuffling word, posed in [5], remained open. We give a positive answer to this question in Sections 2 and 3.

The shift orbit closure $S_{w}$ of an infinite word $w$ can be defined, e.g., as the set of infinite words whose sets of factors are contained in the set of factors of $w$. In [2] it has been proved that each word has a non-self-shuffling word in its shift orbit closure, and the following question has been asked: Does there exist a word for which no element of its shift orbit closure is self-shuffling (Question 7.2)? In Section 4 we provide a positive answer to the question. More generally, we show the existence of a word such that for any three words $x, y, z$ in its shift orbit closure, if $x$ is a shuffle of $y$ and $z$, then the three words are pairwise different. On the other hand, we show that for any infinite word there exist three different words $x, y, z$ in its shift orbit closure such that $x \in \mathscr{S}(y, z)$ (see Proposition 7).

Apart from the usual concepts in combinatorics on words, which can be found for instance in the book of Lothaire [7], we make use of the following notations: For every $k \geqslant 1$, we denote the alphabet $\{0,1, \ldots, k-1\}$ by $\Sigma_{k}$. For a word $w=u v z$ we say that $u$ is a prefix of $w, v$ is a factor of $w$, and $z$ is a suffix of $w$. We denote these prefix-and suffix relations by $u \leqslant_{p} w$ and $v \leqslant_{s} w$, respectively. By $w[i, j]$ we denote the factor of $w$ starting at position $i$ and ending after position $j$. Note that we start numbering the positions with 0 .

A prefix code is a set of words with the property that none of its elements is a prefix of another element. Similarly, a suffix code is a set of words where no element is a suffix of another one. A bifix code is a set that is both a prefix code and a suffix code. A morphism $h$ is square-free if for all square-free words $w$, the image $h(w)$ is square-free.

\section{A square-free self-shuffling word on four letters}

Let $g: \Sigma_{4}^{*} \rightarrow \Sigma_{4}^{*}$ be the morphism defined as follows:

$$
\begin{aligned}
& g(0)=0121, \\
& g(1)=032, \\
& g(2)=013, \\
& g(3)=0302 .
\end{aligned}
$$


We will show that the fixed point $w=g^{\omega}(0)$ is square-free and self-shuffling. Note that $g$ is not a square-free morphism, that is, it does not preserve square-freeness, as $g(23)=0130302$ contains the square 3030 .

Lemma 1. The word $w=g^{\omega}(0)$ contains no factor of the form $3 u 1 u 3$ for any $u \in \Sigma_{4}^{*}$.

Proof. We assume that there exists a factor of the form $3 u 1 u 3$ in $w$, for some word $u \in \Sigma_{4}^{*}$. From the definition of $g$, we observe that $u$ can not be empty. Furthermore, we see that every 3 in $w$ is preceded by either 0 or 1 . If $1 \leqslant_{s} u$, then we had an occurrence of the factor $11 \mathrm{in} w$, which is not possible by the definition of $g$, hence $0 \leqslant_{s} u$. Now, every 3 is followed by either 0 or 2 in $w$ and 01 is followed by either 2 or 3 . Since both $3 u$ and $01 u$ are factors of $w$, we must have $2 \leqslant_{p} u$. This means that the factor 012 appears at the center of $u 1 u$, which can only be followed by 1 in $w$, thus $21 \leqslant_{p} u$. However, this results in the factor 321 as a prefix of $3 u 1 u 3$, which does not appear in $w$, as seen from the definition of $g$.

Lemma 2. The word $w=g^{\omega}(0)$ is square-free.

Proof. We first observe that $\{g(0), g(1), g(2), g(3)\}$ is a bifix code. Furthermore, we can verify that there are no squares $u u$ with $|u| \leqslant 3$ in $w$. Let us assume now, that the square $u u$ appears in $w$ and that $u$ is the shortest word with this property. If $u=02 u^{\prime}$, then $u^{\prime}=u^{\prime \prime} 03$ must hold, since 02 appears only as a factor of $g(3)$, and thus $u u$ is a suffix of the factor $g(3) u^{\prime \prime} g(3) u^{\prime \prime} 03$ in $w$. As $w=g(w)$, also the shorter square $3 g^{-1}\left(u^{\prime \prime}\right) 3 g^{-1}\left(u^{\prime \prime}\right)$ appears in $w$, a contradiction. The same desubstitution principle also leads to occurrences of shorter squares in $w$ if $u=x u^{\prime}$ and $x \in\{01,03,10,12,13,21,30,32\}$.

If $u=2 u^{\prime}$ then either $03 \leqslant_{s} u$ or $030 \leqslant_{s} u$ or $01 \leqslant_{s} u$, by the definition of $g$. In the last case, that is when $01 \leqslant_{s} u$, we must have $21 \leqslant_{p} u$, which is covered by the previous paragraph. If $u^{\prime}=u^{\prime \prime} 030$, then $u u$ is followed by 2 in $w$ and we can desubstitute to obtain the shorter square $g^{-1}\left(u^{\prime \prime}\right) 3 g^{-1}\left(u^{\prime \prime}\right) 3$ in $w$. If $u=2 u^{\prime}$ and $u^{\prime}=u^{\prime \prime} 03$, and $u u$ is preceded by 03 or followed by 2 in $w$, we can desubstitute to $1 g^{-1}\left(u^{\prime \prime}\right) 1 g^{-1}\left(u^{\prime \prime}\right)$ or $g^{-1}\left(u^{\prime \prime}\right) 1 g^{-1}\left(u^{\prime \prime}\right) 1$, respectively. Therefore, assume that $u=2 u^{\prime \prime} 03$ and as we already ruled out the case when $21 \leqslant p u$, we can assume that $u u$ is preceded by 030 and followed by $02 \mathrm{in} w$. This however means that we can desubstitute to get an occurrence of the factor $3 g^{-1}\left(u^{\prime \prime}\right) 1 g^{-1}\left(u^{\prime \prime}\right) 3$ in $w$, a contradiction to Lemma 1.

We now show that $w=g^{\omega}(0)$ can be written as $w=\prod_{i=0}^{\infty} U_{i} V_{i}=\prod_{i=0}^{\infty} U_{i}=\prod_{i=0}^{\infty} V_{i}$ with $U_{i}, V_{i} \in \Sigma_{4}^{*}$.

Lemma 3. The word $w=g^{\omega}(0)$ is self-shuffling.

Proof. We use the notation $x=v^{-1} u$ meaning that $u=v x$ for finite words $x, u, v$. We are going to show that the self-shuffle is given by the following: 


$$
\begin{aligned}
& U_{0}=g^{2}(0), \quad U_{1}=0, \quad \ldots, \quad U_{6 i+2}=g^{i}\left(0^{-1} g(0) 0\right), \quad U_{6 i+3}=g^{i}\left(0^{-1} g(3) 0\right), \\
& U_{6 i+4}=g^{i}\left(0^{-1} g(201) 0\right), \quad U_{6 i+5}=g^{i}(30), \\
& U_{6 i+6}=g^{i}(2 g(03)), \quad U_{6 i+7}=g^{i+1}(20), \\
& V_{0}=g(0) 03, \quad V_{1}=2 g(2) 0, \quad \ldots, \quad V_{6 i+2}=g^{i}\left(0^{-1} g(1) 0\right), \quad V_{6 i+3}=g^{i}\left(0^{-1} g(03) 0\right), \\
& V_{6 i+4}=g^{i}(1), \quad V_{6 i+5}=g^{i}(3), \\
& V_{6 i+6}=g^{i+1}(0), \quad V_{6 i+7}=g^{i+1}\left(0^{-1} g(2) 0\right) \text {. }
\end{aligned}
$$

Now we verify that

$$
w=\prod_{i=0}^{\infty} U_{i} V_{i}=\prod_{i=0}^{\infty} U_{i}=\prod_{i=0}^{\infty} V_{i},
$$

from which it follows that $w$ is self-shuffling. It suffices to show that each of the above products is fixed by $g$. Indeed, straightforward computations show that

$$
\prod_{i=0}^{\infty} U_{i}=g^{2}(0) g^{2}(121) g^{3}(121) \cdots
$$

which is fixed by $g$ :

$$
\begin{aligned}
g\left(\prod_{i=0}^{\infty} U_{i}\right) & =g\left(g^{2}(0) g^{2}(121) g^{3}(121) \cdots\right)=g^{3}(0) g^{3}(121) g^{4}(121) \cdots \\
& =g^{2}(0121) g^{3}(121) g^{4}(121) \cdots=g^{2}(0) g^{2}(121) g^{3}(121) \cdots=\prod_{i=0}^{\infty} U_{i},
\end{aligned}
$$

hence $\prod_{i=0}^{\infty} U_{i}$ is fixed by $g$ and thus $w=\prod_{i=0}^{\infty} U_{i}$. In a similar way we show that $w=\prod_{i=0}^{\infty} V_{i}=\prod_{i=0}^{\infty} U_{i} V_{i}$.

\section{Square-free self-shuffling words on three letters}

We remark that we can immediately produce a square-free self-shuffling word over $\Sigma_{3}$ from $g^{\omega}(0)$ : Charlier et al. [2] noticed that the property of being self-shuffling is preserved by the application of a morphism. Furthermore, Brandenburg [1] showed that the morphism $f: \Sigma_{4}^{*} \rightarrow \Sigma_{3}^{*}$, defined by

$$
\begin{aligned}
& f(0)=010201202101210212, \\
& f(1)=010201202102010212, \\
& f(2)=010201202120121012, \\
& f(3)=010201210201021012,
\end{aligned}
$$

is square-free. Therefore, the word $f\left(g^{\omega}(0)\right)$ is a ternary square-free self-shuffling word, from which we can produce a multitude of others by applying square-free morphisms from $\Sigma_{3}^{*}$ to $\Sigma_{3}^{*}$. 


\section{A word with non self-shuffling shift orbit closure}

In this section we provide a positive answer to the question from [2] whether there exists a word for which no element of its shift orbit closure is self-shuffling.

The Hall word $\mathcal{H}=012021012102 \cdots$ is defined as the fixed point of the morphism $h(0)=012, h(1)=02, h(2)=1$. Sometimes it is referred to as a ternary Thue-Morse word. It is well known that this word is square-free. We show that no word in the shift orbit closure $S_{\mathcal{H}}$ of the Hall word is self-shuffling. More generally, we show that if $x$ is a shuffle of $y$ and $z$ for $x, y, z \in S_{\mathcal{H}}$, then they are pairwise different.

Proposition 4. There are no words $x, y$ in the shift orbit closure of the Hall word such that $x \in \mathscr{S}(y, y)$.

Proof. Suppose the converse, i.e., there exist words $x, y \in S_{\mathcal{H}}$ such that

$$
x=\prod_{i=0}^{\infty} U_{i} V_{i}, \quad y=\prod_{i=0}^{\infty} U_{i}=\prod_{i=0}^{\infty} V_{i} .
$$

Define the set $X$ of infinite words as follows:

$$
X=\left\{x \in S_{\mathcal{H}} \mid x \in \mathscr{S}(y, y) \text { for some } y \in S_{\mathcal{H}}\right\} .
$$

In other words, $X$ consists of words in $S_{\mathcal{H}}$ which can be introduced as a shuffle of some word $y$ in $S_{\mathcal{H}}$ with itself. Now suppose, for the sake of contradiction, that $X$ is non empty, and consider $x \in X$ with the first block $U_{0}$ of the smallest possible positive length. We remark that such $x$ and corresponding $y$ are not necessarily unique.

We can suppose without loss of generality that $y$ starts with 0 or 10 . Otherwise, we exchange 0 and 2 , consider the morphism $0 \mapsto 1,1 \mapsto 20,2 \mapsto 210$, and the argument is symmetric.

It is not hard to see from the properties of the morphism $h$ that removing every occurrence of 1 from $x$ and $y$ results in $(02)^{\omega}$. Hence the blocks in the factorizations of $y$ after removal of 1 are of the form $(02)^{i}$ for some integer $i$. Thus the first letter of each block $U_{i}$ and $V_{i}$ that is different from 1 is 0 , and the last letter different from 1 is 2 .

Then, $U_{i}$ and $V_{i}$ are images by the morphism $h$ of factors of the fixed point of $h$. Therefore, there are words $x^{\prime}, y^{\prime} \in S_{\mathcal{H}}$ such that $x=h\left(x^{\prime}\right), y=h\left(y^{\prime}\right), U_{i}=h\left(U_{i}^{\prime}\right), V_{i}=$ $h\left(V_{i}^{\prime}\right)$, and $x^{\prime}=\prod_{i=0}^{\infty} U_{i}^{\prime} V_{i}^{\prime}, y^{\prime}=\prod_{i=0}^{\infty} U_{i}^{\prime}=\prod_{i=0}^{\infty} V_{i}^{\prime}$.

Notice that the first block $U_{0}$ cannot be equal to 1 . Indeed, otherwise $x$ starts with 11, which is impossible, since 11 is not a factor of the fixed point of $h$.

Clearly, taking the preimage decreases the lengths of blocks in the factorization (except for those equal to 1 ), and since $U_{0} \neq 1$, the length of the first block in the preimage is smaller, i.e., $\left|U_{0}^{\prime}\right|<\left|U_{0}\right|$. This is a contradiction with the minimality of $\left|U_{0}\right|$.

Corollary 5. There are no self-shuffling words in the shift orbit closure of $\mathcal{H}$.

With a similar argument we can prove the following: 
Proposition 6. There are no words $x, y$ in the shift orbit closure of $\mathcal{H}$ such that $x \in$ $\mathscr{S}(x, y)$.

Proof. First we introduce a notation $x \in \mathscr{S}_{2}(y, z)$, meaning that there exists a shuffle starting with the word $z$ (i.e., $U_{0}=\varepsilon, V_{0} \neq \varepsilon$ ). Next, $x \in \mathscr{S}(x, y)$ implies that there exists $z$ in the same shift orbit closure such that $z \in \mathscr{S}_{2}(z, y)$. Indeed, one can remove the prefix $U_{0}$ of $x$ to get $z$, i.e., $z=\left(U_{0}\right)^{-1} x$, and keep all the other blocks $U_{i}, V_{i}$ in the shuffle product.

Define the set $Z$ of infinite words as follows:

$$
Z=\left\{z \in S_{\mathcal{H}} \mid z \in \mathscr{S}_{2}(z, y) \text { for some } y \in S_{\mathcal{H}}\right\}
$$

In other words, $Z$ consists of words in $S_{\mathcal{H}}$ which can be introduced as a shuffle of some word $y$ in $S_{\mathcal{H}}$ with $z$ starting with the block $V_{0}$. Now consider $z \in Z$ with the first block $V_{0}$ of the smallest possible length. We remark that such $z$ and a corresponding $y$ are not necessarily unique.

As in the proof of Proposition 4, the shuffle cannot start with a block of length 1. Again, if we remove every occurrence of 1 in $y$ (and in $z$ ), we get $(02)^{\omega}$ or $(20)^{\omega}$; moreover, since $V_{0}$ contains letters different from 1 , the first letter different from 1 is the same in $y$ and $z$. So, without loss of generality we assume that both $y$ and $z$ without 1 are $(02)^{\omega}$, and the blocks $U_{i}$ and $V_{i}$ without 1 are integer powers of 02 . Then, $U_{i}$ and $V_{i}$ are images by the morphism $h$ of factors of $\mathcal{H}$. Therefore, there are words $z^{\prime}, y^{\prime} \in S_{\mathcal{H}}$ such that $z=h\left(z^{\prime}\right), y=h\left(y^{\prime}\right), U_{i}=h\left(U_{i}^{\prime}\right), V_{i}=h\left(V_{i}^{\prime}\right)$, and $z^{\prime}=\prod_{i=0}^{\infty}\left(U_{i}^{\prime} V_{i}^{\prime}\right)=\prod_{i=0}^{\infty} V_{i}^{\prime}$, $y^{\prime}=\prod_{i=0}^{\infty} U_{i}^{\prime}\left(\right.$ i.e., $z^{\prime} \in Z$ ).

As in the proof of Proposition 4, since $V_{0} \neq 1$, the length of the first block in the preimage is smaller, i.e., $\left|V_{0}^{\prime}\right|<\left|V_{0}\right|$. This is again a contradiction with the minimality of $\left|V_{0}\right|$.

So, we proved that if there are three words $x, y, z$ in the shift orbit closure of the fixed point of $h$ such that $x \in \mathscr{S}(y, z)$, then they should be pairwise distinct. Now we are going to prove that for any infinite word there exist three different words in its shift orbit closure such that $x \in \mathscr{S}(y, z)$.

An infinite word $x$ is called recurrent, if each of its prefixes occurs infinitely many times in it.

Proposition 7. Let $x$ be a recurrent infinite word. Then there exist two words $y, z$ in the shift orbit closure of $x$ such that $x \in \mathscr{S}(y, z)$.

Proof. We build the shuffle inductively.

Start from any prefix $U_{0}$ of $x$. Since $x$ is recurrent, each of its prefixes occurs infinitely many times in it. Find another occurrence of $U_{0}$ in $x$ and denote its position by $i_{1}$. Put $V_{0}=x\left[\left|U_{0}\right|, i_{1}+\left|U_{0}\right|-1\right]$. 
At step $k$, suppose that the shuffle of the prefix of $x$ is built:

$$
\begin{aligned}
x\left[0, \Sigma_{l=0}^{k-1}\left(\left|U_{l}\right|+\left|V_{l}\right|\right)-1\right] & =\prod_{l=0}^{k-1} U_{l} V_{l}, \\
y\left[0, \Sigma_{l=0}^{k-1}\left|U_{l}\right|-1\right] & =\prod_{l=0}^{k-1} U_{l}, \\
z\left[0, \Sigma_{l=0}^{k-1}\left|V_{l}\right|-1\right] & =\prod_{l=0}^{k-1} V_{l},
\end{aligned}
$$

such that $\prod_{l=0}^{k-1} U_{l}$ is the suffix of $x\left[0, \Sigma_{l=0}^{k-1}\left(\left|U_{l}\right|+\left|V_{l}\right|\right)-1\right]=\prod_{i=0}^{k-1} U_{l} V_{l}$ starting at position $i_{k-1}$, and $\prod_{l=0}^{k-1} V_{l}$ is the suffix of $x\left[0, \Sigma_{l=0}^{k-1}\left(\left|U_{l}\right|+\left|V_{l}\right|\right)-1\right]=\prod_{i=0}^{k-1} U_{l} V_{l}$ starting at position $j_{k-1}$.

Find another occurrence of $\prod_{l=0}^{k-1} V_{l}$ in $x$ at some position $j_{k}>j_{k-1}$. We can do it since $x$ is recurrent. Put $U_{k}=x\left[\sum_{l=0}^{k-1}\left(\left|U_{l}\right|+\left|V_{l}\right|\right), j_{k}-1+\sum_{l=0}^{k-1}\left|V_{l}\right|\right]$. We note that $\prod_{l=0}^{k} U_{l}$ is a factor of $x$ by the construction; more precisely, it occurs at position $i_{k-1}$.

Find an occurrence of $\prod_{l=0}^{k} U_{l}$ at some position $i_{k}>i_{k-1}$, put $V_{k}=x\left[\sum_{l=0}^{k-1}\left(\left|U_{l}\right|+\left|V_{l}\right|\right)+\right.$ $\left.\left|U_{k}\right|, i_{k}-1+\sum_{l=0}^{k}\left|U_{l}\right|\right]$. As above, $\prod_{l=0}^{k} V_{l}$ is a factor of $x$ by the construction since it occurs at position $j_{k-1}$. Moreover, both $\prod_{l=0}^{k} U_{l}$ and $\prod_{l=0}^{k} V_{l}$ are suffixes of $x\left[0, \Sigma_{l=0}^{k}\left(\left|U_{l}\right|+\left|V_{l}\right|\right)-\right.$ 1] $=\prod_{i=0}^{k} U_{l} V_{l}$.

Continuing this line of reasoning, we build the required factorization.

Since each infinite word contains a recurrent (actually, even a uniformly recurrent) word in its shift orbit closure, we obtain the following corollary:

Corollary 8. Each infinite word $w$ contains words $x, y, z$ in its shift orbit closure such that $x \in \mathscr{S}(y, z)$.

The following example shows that the recurrence condition in Proposition 7 cannot be omitted:

Example 9. Consider the word $3 \mathcal{H}=3012021 \cdots$ which is obtained from $\mathcal{H}$ by adding a letter 3 in the beginning. Then the shift orbit closure of $3 \mathcal{H}$ consists of the shift orbit closure of $\mathcal{H}$ and the word $3 \mathcal{H}$ itself. Assuming $3 \mathcal{H}$ is a shuffle of two words in its shift orbit closure, one of them is $3 \mathcal{H}$ (there are no other 3's) and the other one is something in the shift orbit closure of $\mathcal{H}$, we let $y$ denote this other word. Clearly, the shuffle starts with 3 , and cutting the first letter 3 , we get $\mathcal{H} \in \mathscr{S}(\mathcal{H}, y)$, a contradiction with Proposition 6 .

There also exist examples where each letter occurs infinitely many times:

Example 10. The following word:

$$
x=012001120001112 \cdots 0^{k} 1^{k} 2 \cdots
$$


does not have two words $y, z$ in its shift orbit closure such that $x \in \mathscr{S}(y, z)$. The idea of the proof is that the shift orbit closure consists of words of the following form: $1^{*} 20^{\omega}$, $0^{*} 1^{\omega}, x$ itself and all their right shifts. Shuffling any two words of those types, it is not hard to see that there exists a prefix of the shuffle which contains too many or too few occurrences of some letter compare to the prefix of $x$. We leave the details of the proof to the reader.

By Corollary 8, there are $x, y, z$ in the shift orbit closure of $\mathcal{H}$ such that $x \in \mathscr{S}(y, z)$. To conclude this section, we give an explicit construction of two words in the shift orbit closure of $\mathcal{H}$ which can be shuffled to give $\mathcal{H}$. We remark though that this construction gives a shuffle different from the one given by Corollary 8. Let:

$$
h:\left\{\begin{aligned}
0 & \mapsto 012 \\
1 & \mapsto 02 \\
2 & \mapsto 1
\end{aligned} \quad \text { and } \quad h^{\prime}:\left\{\begin{aligned}
0 & \mapsto 2210 \\
1 & \mapsto \\
2 & \mapsto 1
\end{aligned}\right.\right.
$$

By definition, the shift orbit closure of the Hall word is closed under $h$. Moreover this shift orbit closure is also closed under $h^{\prime}$. One of the ways to see this is the following. It is well known that the Thue-Morse word, which is a fixed point of the morphism $0 \mapsto 01$, $1 \mapsto 10$ starting with 0 , is a morphic image of $\mathcal{H}$ under a morphism $0 \mapsto 011,1 \mapsto 01$, $2 \mapsto 0$. Therefore, the set of factors of the Hall word is closed under reversal: if $v \in F(\mathcal{H})$, then $v^{R} \in F(\mathcal{H})$ (here for a word $v=v_{1} \cdots v_{n}$ its reversal $v^{R}$ is given by $v^{R}=v_{n} \cdots v_{1}$ ). Now by induction we prove that for each word $v$ one has $h^{\prime}(v)=\left(h\left(v^{R}\right)\right)^{R}$ (it is enough to prove this equality for letters and for the concatenation of two words). This implies that the shift orbit closure of the Hall word is closed $h^{\prime}$.

The morphisms $h$ and $h^{\prime}$ satisfy:

$$
\begin{aligned}
& h^{\prime} \circ h:\left\{\begin{array}{lll}
0 & \mapsto 2 & 210201 \\
1 & \mapsto & 2101 \\
2 & \mapsto 20
\end{array} \quad h \circ h^{\prime}: \begin{cases}0 & \mapsto 102012 \\
1 & \mapsto 1012 \\
2 & \mapsto 002\end{cases} \right. \\
& h^{2}:\left\{\begin{array}{lll}
0 & \mapsto & 012021 \\
1 & \mapsto & 0121 \\
2 & \mapsto & 02
\end{array} \quad h^{\prime 2}:\left\{\begin{array}{lll}
0 & \mapsto & 120210 \\
1 & \mapsto & 1210 \\
2 & \mapsto & 20 .
\end{array}\right.\right.
\end{aligned}
$$

Note that if $w$ is an infinite word, then $2\left(h \circ h^{\prime}\right)(w)=\left(h^{\prime} \circ h\right)(w)$ and $0 h^{\prime 2}(w)=h^{2}(w)$.

Theorem 11. $h^{\omega}(0) \in \mathscr{S}\left(h^{2}\left(\left(h^{\prime 2}\right)^{\omega}(1)\right), h^{\prime 3}\left(h^{\omega}(0)\right)\right)$.

Proof. Let

$$
U_{0}=01, U_{1}=h^{\prime}(0), U_{2}=h^{\prime}(1), V_{0}=h^{\prime}(1)
$$

and for every $i \geqslant 0$,

$$
U_{i+3}=h^{\prime 2}\left(h^{i}(1)\right) \text { and } V_{i+1}=h^{\prime 2}\left(h^{i}(1)\right) .
$$

Let furthermore

$$
u=\prod_{i=0}^{\infty} U_{i}, v=\prod_{i=0}^{\infty} V_{i}, \text { and } w=\prod_{i=0}^{\infty} U_{i} V_{i}
$$


We show that $w=h^{\omega}(0), u=h^{2}\left(\left(h^{\prime 2}\right)^{\omega}(1)\right)$ and $v=h^{\prime 3}\left(h^{\omega}(0)\right)$.

Note that $2 h\left(h^{\prime}\left(h^{\omega}(0)\right)\right)=h^{\prime}\left(h^{\omega}(0)\right)$, thus $h^{\prime}\left(h^{\omega}(0)\right)=\prod_{i=0}^{\infty} h^{i}(2)$. Then we have

$$
v=20 \prod_{i=0}^{\infty} h^{\prime 2}\left(h^{i}(1)\right)=h^{\prime 2}\left(\prod_{i=0}^{\infty} h^{i}(2)\right)=h^{\prime 3}\left(h^{\omega}(0)\right) .
$$

Moreover,

$$
\begin{aligned}
u & =0121020 \prod_{i=0}^{\infty} h^{\prime 2}\left(h^{i}(1)\right)=01210 h^{\prime 2}\left(\prod_{i=0}^{\infty} h^{i}(2)\right) \\
& =01210 h^{\prime 3}\left(h^{\omega}(0)\right)=01 h^{\prime}\left(0 h^{\prime 2}\left(h^{\omega}(0)\right)\right)=01 h^{\prime}\left(h^{\omega}(0)\right)=0 h^{\prime}\left(2 h^{\omega}(0)\right) .
\end{aligned}
$$

Since $h^{\prime 2}\left(2 h^{\omega}(0)\right)=20 h^{\prime 2}\left(h^{\omega}(0)\right)=2 h^{\omega}(0)$, the word $2 h^{\omega}(0)$ is the fixed point $\left(h^{\prime 2}\right)^{\omega}(2)$ of $h^{\prime 2}$, and then $h^{\prime}\left(2 h^{\omega}(0)\right)$ is the fixed point $\left(h^{\prime 2}\right)^{\omega}(1)$. Thus $u=0\left(h^{\prime 2}\right)^{\omega}(1)=h^{2}\left(\left(h^{\prime 2}\right)^{\omega}(1)\right)$. Finally:

$$
w=0120210121020 \prod_{i=0}^{\infty} h^{\prime 2}\left(h^{i}(021)\right)=012021 h(021) h^{2}\left(\prod_{i=0}^{\infty} h^{i}(021)\right)=012 \prod_{i=0}^{\infty} h^{i}(021) .
$$

Applying the morphism $h$ to the second expression for $w$, we get

$$
h(w)=012021 h\left(\prod_{i=0}^{\infty} h^{i}(021)\right)=012 \prod_{i=0}^{\infty} h^{i}(021) .
$$

Thus $w=h^{\omega}(0)$ since $h$ is injective.

\section{Conclusions}

We showed that infinite square-free self-shuffling words exist. The natural question that arises now is whether we can find infinite self-shuffling words subject to even stronger avoidability constraints: For this we recall the notion of repetition threshold $R T(k)$, which is defined as the least real number such that an infinite word over $\Sigma_{k}$ exists, that does not contain repetitions of exponent greater than $R T(k)$. Due to the collective effort of many researchers (see $[3,8]$ and references therein), the repetition threshold for all alphabet sizes is known and characterized as follows:

$$
R T(k)= \begin{cases}\frac{7}{4} & \text { if } k=3 \\ \frac{7}{5} & \text { if } k=4 \\ \frac{k}{k-1} & \text { else. }\end{cases}
$$

A word $w \in \Sigma_{k}^{\omega}$ without factors of exponent greater than $R T(k)$ is called a Dejean word. Charlier et al. showed that the Thue-Morse word, which is a binary Dejean word, is self-shuffling [2].

Question 12. Do there exist self-shuffling Dejean words over non-binary alphabets? 


\section{References}

[1] F.-J. Brandenburg. Uniformly Growing $k$ th Power-Free Homomorphisms. Theor. Comput. Sci. 23: 69-82, 1983.

[2] E. Charlier, T. Kamae, S. Puzynina, L. Q. Zamboni. Infinite self-shuffling words. J. Comb. Theory, Ser. A, 128: 1-40, 2014.

[3] J. Currie, N. Rampersad. A proof of Dejean's conjecture. Math. Comput. 80(274): 1063-1070, 2011.

[4] J. Currie, K. Saari. Square-free Words with Square-free Self-shuffles. Electr. J. Comb., 21(1): \#P1.9 (2014).

[5] T. Harju. A Note on Square-Free Shuffles of Words. LNCS 8079 (WORDS 2013): 154-160.

[6] T. Harju, M. Müller. Square-Free Shuffles of Words. CoRR abs/1309.2137 (2013)

[7] M. Lothaire. Algebraic combinatorics on words. Cambridge University Press, 2002.

[8] M. Rao. Last cases of Dejean's conjecture. Theor. Comput. Sci. 412(27): 3010-3018, 2011. 